\title{
Gastric mucosa-associated lymphoid tissue lymphoma with central nervous system involvement: a case report and 8-year follow-up
}

\author{
Haiyu Niu ${ }^{1}$, Hanwen Wei $^{2}$, Feixue Song ${ }^{1}$ \\ ${ }^{1}$ Department of Oncology, Lanzhou University Second Hospital, Lanzhou, China; ${ }^{2}$ Department of Cardiology, The First People's Hospital of \\ Lanzhou, Lanzhou, China \\ Correspondence to: Feixue Song, MD. Department of Oncology, Lanzhou University Second Hospital, Lanzhou 730030, China. \\ Email: 13893273778@139.com.
}

\begin{abstract}
Gastric mucosa-associated lymphoid tissue (MALT) lymphoma is a form of low-grade B cell lymphoma that is associated with Helicobacter pylori (H. pylori) infection and has a generally favorable prognosis. It tends to remain localized for extended periods before dissemination to other body parts. H. pylori eradication therapy is essential in all gastric MALT lymphoma patients regardless of the disease stage. However, no conclusive treatment regimen for gastric MALT lymphoma with central nervous system (CNS) involvement has been established to date. Herein we present a case of a gastric MALT lymphoma patient with CNS involvement who was successfully treated via combination chemoimmunotherapy and intrathecal chemotherapy. A 53-year-old woman was diagnosed with stage IV gastric MALT lymphoma with CNS involvement in 2012. She underwent 6 cycles of R-CHOP (rituximab, cyclophosphamide, doxorubicin, vincristine, and prednisolone), 2 cycles of rituximab, and 10 cycles of intrathecal chemotherapy. Six months later, radiological testing revealed no evidence of disease. In 2019 a mass was discovered in her right parietal lobe. She again underwent 6 R-CHOP cycles and 8 intrathecal chemotherapy cycles. The patient is being actively followed without any evidence of recurrence. Based on this successful case, chemoimmunotherapy combined with intrathecal chemotherapy could possibly be used for the treatment of gastric MALT lymphoma with CNS involvement.
\end{abstract}

Keywords: Gastric; mucosa-associated lymphoid tissue lymphoma (MALT lymphoma); central nervous system (CNS); chemoimmunotherapy; case report

Submitted Mar 17, 2021. Accepted for publication Jul 16, 2021.

doi: $10.21037 /$ tcr-21-473

View this article at: https://dx.doi.org/10.21037/tcr-21-473

\section{Introduction}

Gastric mucosa-associated lymphoid tissue (MALT) lymphoma is a form of low-grade B cell lymphoma that is associated with Helicobacter pylori infection and has a generally favorable prognosis (1). The age-standardized incidence rates of MALT-lymphoma among males and females in Korea were 1.53 and 1.61 per 100,000, respectively, in 2017, compared to 0.21 and 0.20 in 1999 (2). According to a recent study, the incidence of gastric MALT lymphoma in the United States was around 0.38 per 100,000 people, with rates rising in direct proportion to age (3). Almost $10 \%$ of low-grade gastric MALT lymphoma are in advanced stages (III-IV) and require anti-neoplastic therapy (1). The symptoms of gastric MALT lymphoma are usually non-specific and include weight loss, dyspepsia, nausea, vomiting, and epigastric pain that may be attributed to gastric ulcers or other tumor types. Patients rarely experience disease-related complications such as bleeding, perforation, or obstruction $(4,5)$, and the disease is generally detected at an early stage, with the advanced disease being rarely reported. In the present report, however, we describe the case of a patient with gastric MALT lymphoma 

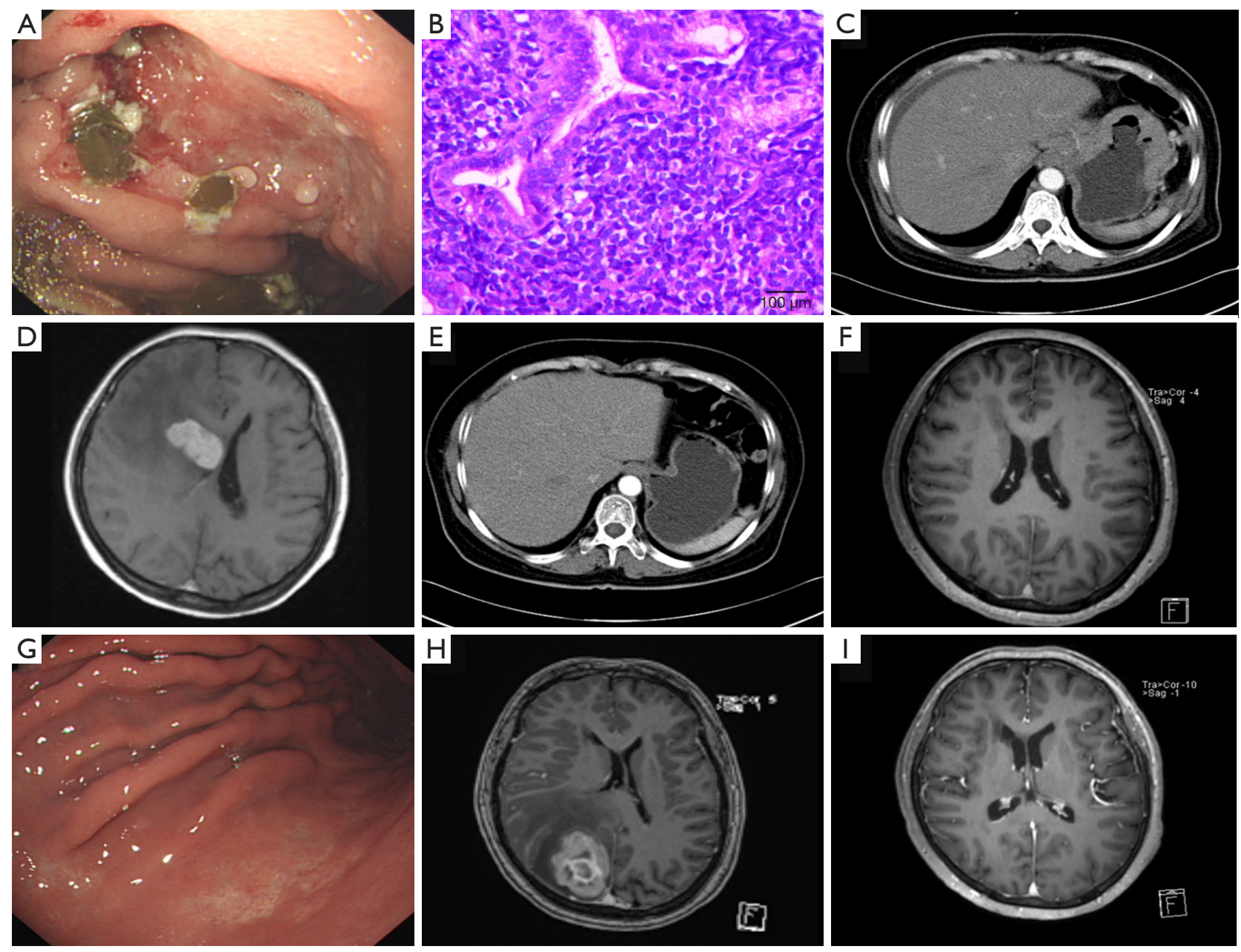

Figure 1 Imaging results from a 53-year-old female patient affected by gastric MALT lymphoma with CNS involvement. (A) Endoscopic imaging revealed the presence of an ulcer in the gastric body. (B) HE staining exhibiting diffuse CCL cell infiltration and LELs ( $\times 400$ ). (C) Abdominal CT scan images revealing diffuse thickening of the stomach wall. (D) MRI scan results revealing a mass in the right basal ganglia region with peritumoral edema. (E) Abdominal CT scan results indicating the presence of a largely normal gastric wall. (F) MRI results highlighting the disappearance of the mass in the right basal ganglia region. $(\mathrm{G})$ Endoscopic images exhibiting gastric ulcer scars. (H) MRI scan results demonstrating the presence of a mass in the right parietal lobe with peritumoral edema. (I) MRI scan results highlighting the disappearance of the mass in the right parietal lobe. MALT, mucosa-associated lymphoid tissue; CNS, central nervous system; CCL, centrocyte-like; LELs, lymphoepithelial lesions; CT, computed tomography; MRI, magnetic resonance imaging.

exhibiting central nervous system (CNS) involvement. We present the following article in accordance with the CARE reporting checklist (available at https://dx.doi.org/10.21037/ tcr-21-473).

\section{Case presentation}

In May 2012, a 53-year-old woman came to our hospital with hematemesis, melena, and dizziness, and she was treated with intravenous proton-pump inhibitor therapy. Endoscopic examination revealed the presence of multiple ulcers in the fundus, gastric body, and antrum (Figure 1A). Biopsy samples revealed the presence of small centrocytelike (CCL) cells forming lymphoepithelial lesions (LELs) (Figure $1 B$ ), and immunohistochemical staining of these cells revealed them to be positive for CD20 expression but negative for CD5, CD10, and cyclin D1 expression. Abdominal computed tomography (CT) imaging revealed 
the diffuse thickening of the stomach wall and the presence of several abdominal masses (Figure 1C). Cranial magnetic resonance imaging (MRI) revealed the presence of a mass in the right basal ganglia region that was deemed secondary to primary gastric lymphoma (Figure 1D). Subsequent positron emission tomography (PET)-CT scan conducted at another hospital revealed the increased uptake of fludeoxyglucose in the stomach, the thoracic and abdominal lymph nodes, and the right basal ganglia mass. The patient was diagnosed with stage IV gastric MALT lymphoma (Lugano staging system) associated with CNS and multiple lymph node involvements (6), and declined the opportunity to undergo invasive brain surgery.

Chemoimmunotherapy in this patient was subsequently initiated, consisting of 6 cycles of R-CHOP (rituximab, cyclophosphamide, doxorubicin, vincristine, and prednisolone), 2 cycles of rituximab, and 10 cycles of intrathecal chemotherapy (dexamethasone, methotrexate, and cytosine arabinoside). At 6 months post-treatment, no obvious gastric wall abnormalities or lymph node enlargement were detected in chest, abdomen, and pelvis CT scans (Figure 1E), and the cranial mass was no longer evident upon MRI evaluation (Figure $1 F$ ). Endoscopy revealed the presence of gastric ulcer scars (Figure 1G). The patient continued to undergo active clinical and radiological surveillance without any evidence of disease recurrence but declined the opportunity to undergo additional endoscopic examination.

In February 2019, the patient again presented with dizziness. As relapse was suspected, an endoscopic examination was conducted that revealed a pattern consistent with chronic gastritis. Chest, abdomen, and pelvis CT scans excluded local recurrence, but an MRI revealed the presence of a mass in the right parietal lobe (Figure 1H). The patient underwent 6 chemoimmunotherapy cycles of R-CHOP and 8 cycles of intrathecal chemotherapy (dexamethasone, methotrexate, and cytosine arabinoside). A chest, abdominal, and pelvis CT scan five months after therapy found no sign of recurrence, while cranial MRI scan revealed that the mass in the right parietal lobe had vanished (Figure 1I). The patient is currently undergoing active follow-up without any evidence of disease recurrence.

All procedures performed in studies involving human participants were in accordance with the ethical standards of the institutional and/or national research committee(s) and with the Helsinki Declaration (as revised in 2013). Written informed consent was obtained from the patient.

\section{Discussion}

MALT lymphoma accounts for 7-8\% of all non-Hodgkin's lymphoma cases (7), manifesting at different extranodal sites, with the stomach being the most common (70\%) (8). Few studies reported the involvement of CNS in MALT lymphoma $(9,10)$. The present case is a relatively rare report of gastric MALT lymphoma with CNS involvement.

Helicobacter pylori eradication therapy is essential in all gastric MALT lymphoma patients regardless of disease stage (11). A significant number of patients with HPnegative gastric MALT responded to first-line Helicobacter pylori therapy $(12,13)$. When antibiotic therapy is insufficient to achieve disease regression, irradiation and systemic antitumor treatments should be employed as appropriate $(14,15)$. Radiotherapy is generally the preferred treatment for patients with localized disease. As advancedstage disease is not generally curable, it is recommended that affected individuals exhibiting symptoms such as gastrointestinal bleeding and early satiety should enroll in clinical trials where possible with chemoimmunotherapy being an alternative approach when suitable trials are not available (16). For around 25 years, the cyclophosphamide, doxorubicin, vincristine, and prednisone (CHOP) regimen has been the gold standard for treating patients with diffuse large B-cell lymphoma (DLBCL). Several studies have found that adding rituximab to the $\mathrm{CHOP}$ regimen (R-CHOP) improves complete response rates, decreases relapse rates, and improves event-free survival (EFS) and overall survival (OS) (17). No conclusive treatment regimen for gastric MALT lymphoma with CNS involvement has been established to date. Given the good effects of R-CHOP in DLBCL and the same origin of DLBCL and gastric MALT lymphoma, we attempted an R-CHOP regimen for treating this patient. As a result, the patient was successfully treated. Therefore, chemoimmunotherapy combined with intrathecal chemotherapy could possibly be used for the treatment of this cancer type.

MALT lymphoma is an indolent B-cell lymphoma characterized by a fascinating interplay between chronic antigenic stimulation, and immune response insufficient for the elimination of the antigen and a mucosal 'battleground'. Therefore, it may have the possibility of relapse (18). In case of relapse, various therapies such as targeted therapies (novel agents targeting BTK and Pi3K) or the use of immunomodulatory agents may be considered to tackle the situation. For instance, due to the role of the NF-kB pathway in the pathogenesis of MALT lymphoma, the 
proteasome inhibitor bortezomib can be considered to treat the MALT (18). Given the dependency of MALT lymphoma cells on the tumor microenvironment, this specific entity appears highly suitable for immunomodulatory treatment strategies (19).

In summary, we herein presented the case of a gastric MALT lymphoma patient with CNS involvement who was successfully treated via combination chemoimmunotherapy and intrathecal chemotherapy.

\section{Acknowledgments}

Funding: This work was supported by grants from the Innovation and Entrepreneurship Talents Project of Longyuan Youth (2020RCXM127).

\section{Footnote}

Reporting Checklist: The authors have completed the CARE reporting checklist. Available at https://dx.doi. org/10.21037/tcr-21-473

Peer Review File: Available at https://dx.doi.org/10.21037/ tcr-21-473

Conflicts of Interest: All authors have completed the ICMJE uniform disclosure form (available at https://dx.doi. org/10.21037/tcr-21-473). The authors have no conflicts of interest to declare.

Ethical Statement: The authors are accountable for all aspects of the work in ensuring that questions related to the accuracy or integrity of any part of the work are appropriately investigated and resolved. All procedures performed in studies involving human participants were in accordance with the ethical standards of the institutional and/or national research committee(s) and with the Helsinki Declaration (as revised in 2013). Written informed consent was obtained from the patient for publication of this case report and accompanying images. A copy of the written consent is available for review by the editorial office of this journal.

Open Access Statement: This is an Open Access article distributed in accordance with the Creative Commons Attribution-NonCommercial-NoDerivs 4.0 International License (CC BY-NC-ND 4.0), which permits the noncommercial replication and distribution of the article with the strict proviso that no changes or edits are made and the original work is properly cited (including links to both the formal publication through the relevant DOI and the license). See: https://creativecommons.org/licenses/by-nc-nd/4.0/.

\section{References}

1. Zullo A, Hassan C, Ridola L, et al. Gastric MALT lymphoma: old and new insights. Ann Gastroenterol 2014;27:27-33.

2. Jeong SH, Hyun SY, Choi JS, et al. Trends of Incidence and Survival Rates of Mucosa-associated Lymphoid Tissue Lymphoma in the Korean Population: Analysis of the Korea Central Cancer Registry Database. J Korean Med Sci 2020;35:e294.

3. Hu Q, Zhang Y, Zhang X, et al. Gastric mucosa-associated lymphoid tissue lymphoma and Helicobacter pylori infection: a review of current diagnosis and management. Biomark Res 2016;4:15.

4. Ma X, Qin L, Liu Y, et al. Perforation caused by gastric mucosa associated lymphoid tissue lymphoma: A case report and literature review. Medicine (Baltimore) 2018;97:e11713.

5. Violeta Filip P, Cuciureanu D, Sorina Diaconu L, et al. MALT lymphoma: epidemiology, clinical diagnosis and treatment. J Med Life 2018;11:187-93.

6. Juárez-Salcedo LM, Sokol L, Chavez JC, et al. Primary Gastric Lymphoma, Epidemiology, Clinical Diagnosis, and Treatment. Cancer Control 2018;25:1073274818778256.

7. A clinical evaluation of the International Lymphoma Study Group classification of non-Hodgkin's lymphoma. The Non-Hodgkin's Lymphoma Classification Project. Blood 1997;89:3909-18.

8. Isaacson PG, Du MQ. MALT lymphoma: from morphology to molecules. Nat Rev Cancer 2004;4:644-53.

9. Matmati K, Matmati N, Hannun YA, et al. Dural MALT lymphoma with disseminated disease. Hematol Rep 2010;2:e10.

10. Huang HC, Cheng AL, Lin CW, et al. Primary central nervous system diffuse large B cell lymphoma transformed from orbital mucosa-associated lymphoid tissue lymphoma: complete response to combined intrathecal and systemic rituximab. Ann Hematol 2013;92:989-92.

11. Dreyling M, Thieblemont C, Gallamini A, et al. ESMO Consensus conferences: guidelines on malignant lymphoma. part 2: marginal zone lymphoma, mantle cell lymphoma, peripheral T-cell lymphoma. Ann Oncol 
2013;24:857-77.

12. Kuo SH, Yeh KH, Wu MS, et al. First-line antibiotic therapy in Helicobacter pylori-negative low-grade gastric mucosa-associated lymphoid tissue lymphoma. Sci Rep 2017;7:14333.

13. Won JH, Kim SM, Kim JW, et al. Clinical features, treatment and outcomes of colorectal mucosa-associated lymphoid tissue (MALT) lymphoma: literature reviews published in English between 1993 and 2017. Cancer Manag Res 2019;11:8577-87.

14. Martinelli G, Laszlo D, Ferreri AJ, et al. Clinical activity of rituximab in gastric marginal zone non-Hodgkin's lymphoma resistant to or not eligible for anti-Helicobacter pylori therapy. J Clin Oncol 2005;23:1979-83.

15. Tsai $\mathrm{HK}, \mathrm{Li} \mathrm{S}, \mathrm{Ng} \mathrm{AK}$, et al. Role of radiation therapy in the treatment of stage I/II mucosa-associated lymphoid tissue lymphoma. Ann Oncol 2007;18:672-8.

16. Zucca E, Copie-Bergman C, Ricardi U, et al. Gastric marginal zone lymphoma of MALT type: ESMO Clinical Practice Guidelines for diagnosis, treatment and followup. Ann Oncol 2013;24 Suppl 6:vi144-8.

17. Tariq A, Aziz MT, Mehmood Y, et al. Clinical Response to CHOP vs. R-CHOP in Adult Patients with Diffuse Large B-Cell Lymphomas. Asian Pac J Cancer Prev 2018;19:1181-4.

18. Raderer M, Kiesewetter B. How I treat MALT lymphoma: 'a subjective interpretation of the gospel according to Isaacson....' ESMO Open 2020;5:e000812.

19. Kiesewetter B, Raderer M. Immunomodulatory treatment for mucosa-associated lymphoid tissue lymphoma (MALT lymphoma). Hematol Oncol 2020;38:417-24.
Cite this article as: Niu H, Wei H, Song F. Gastric mucosaassociated lymphoid tissue lymphoma with central nervous system involvement: a case report and 8-year follow-up. Transl Cancer Res 2021;10(9):4232-4236. doi: 10.21037/tcr-21-473 